\title{
A hierarchical Dempster-Shafer evidence combination framework for urban area land cover classification
}

Article

Accepted Version

Creative Commons: Attribution-Noncommercial-No Derivative Works 4.0

Yang, F., Wei, H. and Feng, P. (2020) A hierarchical DempsterShafer evidence combination framework for urban area land cover classification. Measurement, 151. 105916. ISSN 02632241 doi: https://doi.org/10.1016/j.measurement.2018.09.058 Available at https://centaur.reading.ac.uk/80424/

It is advisable to refer to the publisher's version if you intend to cite from the work. See Guidance on citing.

To link to this article DOI: http://dx.doi.org/10.1016/j.measurement.2018.09.058

Publisher: Elsevier

All outputs in CentAUR are protected by Intellectual Property Rights law, including copyright law. Copyright and IPR is retained by the creators or other copyright holders. Terms and conditions for use of this material are defined in the End User Agreement.

www.reading.ac.uk/centaur 
Central Archive at the University of Reading

Reading's research outputs online 


\section{Accepted Manuscript}

A hierarchical Dempster-Shafer evidence combination framework for urban area land cover classification

Fengbao Yang, Hong Wei, Peipei Feng

PII:

S0263-2241(18)30894-7

DOI: https://doi.org/10.1016/j.measurement.2018.09.058

Reference: MEASUR 5916

To appear in:

Measurement

Received Date: $\quad 29$ January 2018

Revised Date: $\quad 27$ March 2018

Accepted Date: $\quad 23$ September 2018

Please cite this article as: F. Yang, H. Wei, P. Feng, A hierarchical Dempster-Shafer evidence combination framework for urban area land cover classification, Measurement (2018), doi: https://doi.org/10.1016/ j.measurement.2018.09.058

This is a PDF file of an unedited manuscript that has been accepted for publication. As a service to our customers we are providing this early version of the manuscript. The manuscript will undergo copyediting, typesetting, and review of the resulting proof before it is published in its final form. Please note that during the production process errors may be discovered which could affect the content, and all legal disclaimers that apply to the journal pertain. 


\title{
A hierarchical Dempster-Shafer evidence combination framework for urban area land cover classification
}

Fengbao Yang ${ }^{1}$, Hong Wei ${ }^{1,2}$, Peipei Feng ${ }^{1}$

\author{
${ }^{1}$ School of Information and Communication Engineering, North University of China, Shanxi 030051, China \\ ${ }^{2}$ Department of Computer Science, University of Reading, Reading, RG6 6AY, UK

\section{Corresponding author: Fengbao Yang} \\ Email:yfengb@163.com
}

\begin{abstract}
This paper presents a novel evidence combination framework for urban area land cover classification by using Light Detection And Ranging (LIDAR) data fused with co-registered near infrared and color images. The newly developed combination framework is built with a hierarchical structure involving an improved Dempster-Shafer (DS) theory of evidence for decision making. In the framework, a fuzzy basic probability assignment (BPA) function with fuzzy classes is firstly established based on the DS theory of evidence, and a probability is then assigned to each data source, that is derived from the original airborne LIDAR and the co-registered images. Secondly, an interesting approach is to introduce noise removal in an interim stage at the output of the probability distribution, and then the probability assigned to each data source is redistributed with a designated rule. Finally, a decision is made based on a "maximum normal support" rule, leading to the classification results. The proposed framework has been tested on two datasets. The testing results have shown that it can dramatically reduce the computational time in the classification process, and significantly improve the classification accuracy, i.e. $8.22 \%$ on Test 1 and $5.76 \%$ on Test 2 compared to the basic DS method. Due to its non-iterative and unsupervised nature, the proposed method is fast in computation, does not require training samples, and has achieved high classification accuracy.
\end{abstract}

Keywords: Dempster-Shafer evidence, fuzzy BPA function, LIDAR data, land cover classification

\section{Introduction}

\subsection{Motivation and study goals}

Computational approaches to make use of airborne LIDAR data have made significant contributions to many applications, such as forestry modeling [1], road extraction [2], and 3D building modeling [3]. Usually data acquired by an airborne LIDAR system include height information regarding the land-cover with a high spatial resolution, as well as LIDAR intensity and spectral information, such as visible images and near infrared images. These have made the data perform well in terrain and land surveying. This study is focused on exploration of the capability of LIDAR data, associated with its co-registered spectral images in land-cover classification, especially in an urban area with land cover classes such as building, tree, road and grass. The main requirements for land cover classification are high classification accuracy and low computational cost. In previous studies, researchers focused mainly on improving classification accuracy [4]. Nevertheless acquired LIDAR data become more complex with increasing complexity of landscape to be surveyed and with high resolution point cloud. These demand advanced computational efficiency. For applications such as selecting a bombing target, aircraft landing navigation in extreme weather conditions, and rescuing people during natural disasters, a classification method must satisfy the requirement of not only high accuracy but must also have high computational efficiency. 
Furthermore, these situations may not allow a supervised learning process, in which training is needed. Thus, developing a fast and high precision land cover classification method with available data resources is needed.

A set of co-registered data can be obtained with LIDAR systems, such as LIDAR first echo (FE), LIDAR last echo (LE), LIDAR intensity, aerial images such as red, green, blue (RGB) and near-infrared (NIR) images. A number of supervised classification methods have achieved good classification accuracy with these data, such as the Markov random field method (MRF) or conditional random field method (CRF) [5], support vector machine method (SVM) [6] and random forest method $(\mathrm{RF})[7,8]$. In recent studies with regards to land cover classification from remotely sensed imagery, $\mathrm{Xu}$, et al. used deep learning algorithms in automatic land cover classification [9], Chen, et al. employed SVM as a classifier in their study of multi-source remotely sensed data fusion for improving land cover classification [10], and Pereira, et al adapted contextual learning into land use classification for better representation of their model [11]. However due to the nature of supervised learning, these methods are not only computationally expensive in terms of iterative training, but also require training samples. The study by Niemeyer et al. [5] reported that it took 202 minutes and 252 minutes to train the CRF and MRF, respectively, with a study area of 1.93 ha. Besides this, these approaches require a large amount of detailed knowledge of the land-cover in a scene to start the classification. Yan, et al. summarized the popular classifiers used in urban land cover classification in their review paper [4], and it revealed that the majority of the classifiers belong to supervised learning in which training samples are always needed.

Land cover classification methods are sought after for applications where training samples are not available. Dempster-Shafer (DS) theory of evidence, as a basic method for dealing with problems of uncertainty, requires neither training samples nor priori knowledge of the area in question. Cao et al. [12] employed the DS evidence theory as the initial segmentation algorithm in their study on LIDAR based land cover classification, and the Markov random field model was further combined into the classifier to improve the performance. They used a linear mass function to reduce the impact of uncertainty in the initial classification. However the iterative nature of the MRF algorithm caused an extended computation time in the further classification. Rottensteiner et al. [13] used the DS evidence theory to process a fusion of LIDAR data and multi-spectral images in building detection. A three-times-BPA function was proposed as the mass function, and they obtained better classification results compared to the basic DS method. Nevertheless the BPA function is still a single function without consideration of fuzziness in classification.

In the previous studies $[12,13,14]$, linear mass functions were used to distribute the probability to each class based on given thresholds under the DS evidence theory framework. The linear mass function fails to solve the problem of those uncertain pixels that reside in the region mixed with different classes. In this study, we propose a fuzzy class in the mixed region to classify pixels that do not clearly fit into any classes, hence the distribution of probability values solves the problem of uncertainty over that region. Classification accuracy is improved effectively by integrating the median filtering and a hierarchical combination framework proposed in this study.

The rest of the paper is laid out in the following order. Section 2 introduces the hierarchical Dempster-Shafer (DS) evidence combination framework, as well as features used in the study. The detailed approach and related techniques are presented in Section 3. We demonstrate the experimental results and discuss them in Section 4. Finally Section 5 concludes the study. 


\section{The framework and the feature space}

The source data used in this study are the LIDAR first echo elevation image (FE), the last echo elevation image (LE), intensity image (IN), RGB images and near infrared image (NIR). Other two images, HD and NDVI, are obtained from the following formula:

$$
\begin{gathered}
\text { HD }=\text { FE }- \text { LE } \\
\text { NDVI }=\frac{\text { NIR }- \text { Red }}{\text { NIR }+ \text { Red }}
\end{gathered}
$$

HD is the height difference between the first and the last echo elevation images. It is used to separate trees and buildings according to the penetration characteristics of LIDAR to trees; NDVI, derived from NIR and Red image, is the Normalized Difference Vegetation Index image, which is conventionally used for vegetation identification. In this study, based on the physical properties of the data, FE, IN, HD and NDVI are selected as features to classify land covers into four classes: building $(B)$, grass $(G)$, road $(R)$, and tree $(T)$.

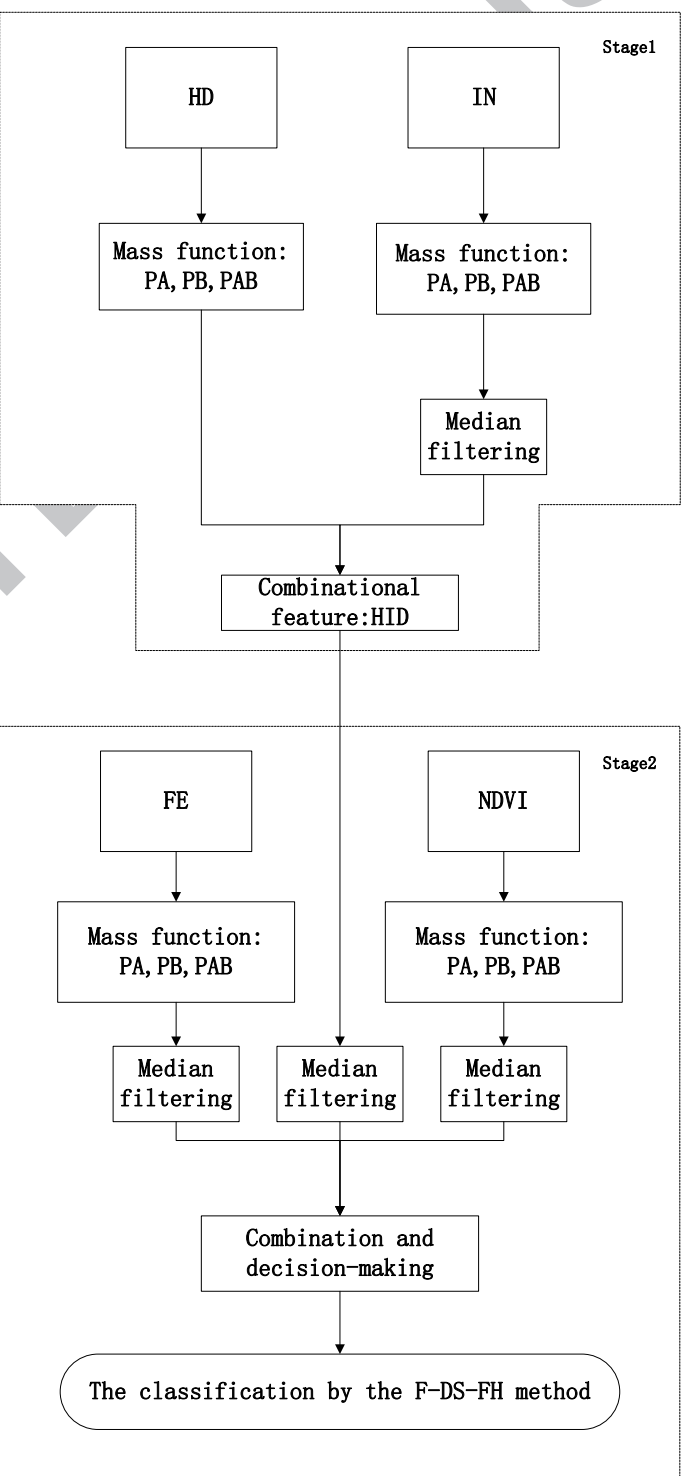

Figure 1. Framework of the land cover classification 
The framework of the land cover classification is demonstrated in Figure 1. With consideration of each feature's characteristics and its contribution to the four classes, instead of applying all features at the same time, a hierarchical framework is established. The process is divided into two stages, and the details are described as follows.

Stage 1: Since HD and IN have complementary functions in identifying trees from other classes observed from our experiments, the framework accepts these two features to separate trees from other classes (including fuzzy classes). First, the mass functions are established according to the basic probability assignment (BPA) function, and the results are expressed as $\mathrm{P}_{\mathrm{A}_{\mathrm{HD}}}, \mathrm{P}_{\mathrm{B}_{\mathrm{HD}}}, \mathrm{P}_{\mathrm{A \cup B}} \mathrm{B}_{\mathrm{HD}}, \mathrm{P}_{\mathrm{A}_{I N}}, \mathrm{P}_{\mathrm{B}_{I N}}$ and $\mathrm{P}_{\mathrm{AUB}_{I N}}$. Next, a median filter is applied to the output of the mass functions of IN, based on an interesting observation showing sparse noise introduced by the process as well as the original data. HID, the output of Stage 1 is the combinational feature of HD and IN, and obtained from a support function through the Dempster-Shafer evidence theory. HID is also one of the three input features to Stage 2.

Stage 2: The inputs of Stage 2 compose of three features FE, NDVI and HID. Mass functions to FE and NDVI are: $\mathrm{P}_{\mathrm{A}_{\mathrm{FE}}}, \mathrm{P}_{\mathrm{AUB}_{\mathrm{FE}}}, \mathrm{P}_{\mathrm{B}_{\mathrm{FE}}}, \mathrm{P}_{\mathrm{A}_{\mathrm{NDVI}}}, \mathrm{P}_{\mathrm{B}_{\mathrm{NDVI}}}$ and $\mathrm{P}_{\mathrm{A} \cup B_{\mathrm{NDVI}}}$. Then median filtering is conducted to reduce noise of mass function outputs of FE, NDVI and HID individually. The parameters of BPA function are different from those used in Stage 1 and the details of choosing parameters are discussed in Section 4. The final classification results are achieved by the DS evidence combination method and decision rules, which are denoted as the F-DS-FH method in Figure 1 and will be explained in details in Section 3.4.

\section{Combination method}

\subsection{Dempster-Shafer evidence theory}

The Dempster-Shafer evidence theory is frequently applied for fusion of data from multiple sensors [15]. Unlike Bayesian probabilistic reasoning, it offers tools to represent partial knowledge about a sensor's contribution to the classification process.

Let us assume a classification problem in which input data are to be classified into mutually exclusive classes. The set $\Theta$ of these classes is called the frame of discernment. $2^{\Theta}$ is a power set of $\Theta$ which contains both the classes and all their possible unions. In Dempster-Shafer theory, a probability mass $m(A)$ is assigned to each class $A \in 2^{\Theta}$ by a sensor such that $0 \leq m(A) \leq 1$,

$m(\varnothing)=0$, and $\sum_{A \in 2^{\Theta}} m(A)=1$, where $\varnothing$ denotes an empty set. $m(A)$ can be computed by a function, i.e. a BPA function, from the data provided by a sensor.

Imprecision of knowledge can be handled by assigning a non-zero probability mass to the union of two or more classes. Two parameters, support $\operatorname{Sup}(A)$ and plausibility Pls $(A)$, can be defined for all $A \in 2^{\Theta}:$

$$
\begin{aligned}
& \operatorname{Sup}(A)=\sum_{B_{\mathrm{S}} \subseteq A} m\left(B_{\mathrm{S}}\right) \\
& \operatorname{Pls}(A)=\sum_{A \cap B_{\mathrm{PL}} \neq \varnothing} m\left(B_{\mathrm{PL}}\right)=1-\operatorname{Sup}(\bar{A})
\end{aligned}
$$

where $B_{\mathrm{S}}, B_{\mathrm{PL}} \in 2^{\Theta}$. The support of a class is the sum of all the probability masses directly assigned 
to that class by a data source, whereas the plausibility is the sum of all the probability masses not assigned to the complement of the class. $\bar{A}$ is the complementary hypothesis of $A . \operatorname{Sup}(\bar{A})$ represents the degree to which the evidence contradicts a proposition.

If $p$ data sources are available, probability masses $m_{i}\left(B_{j}\right)$ have to be defined for each data source $i$ with $0 \leq i \leq p$ and for all classes $B_{j} \in 2^{\Theta}$. Dempster-Shafer theory allows the combination of these probability masses from several data sources to compute a combined probability mass for each class $A \in 2^{\Theta}$ :

$$
m(A)=\frac{\sum_{B_{1} \cap B_{2} \cdots \cap B_{p}=A}\left(\prod_{1 \leq i \leq p} m_{i}\left(B_{j}\right)\right)}{1-\sum_{B_{1} \cap B_{2} \cdots \cap B_{p}=\emptyset}\left(\prod_{1 \leq i \leq p} m_{i}\left(B_{j}\right)\right)}
$$

The sum in the denominator of Equation (3) is a measure of the conflict in the evidence. As soon as the combined probability masses $m(A)$ have been derived from the original masses, both $\operatorname{Sup}(A)$ and $\operatorname{Pls}(A)$ can be computed. Finally, a decision rule must be defined in order to determine the accepted simple hypothesis [16].

There are several ways of defining such a decision rule with hypothesis of (i) maximum support, (ii) maximum plausibility, (iii) maximum support without overlapping of uncertainty intervals, or (iv) maximum normal support. We use the rule of maximum normal support as it is a commonly used rule. The definition of normal support is shown in Equation (4).

$$
M_{n}(A)=\sum_{A \subseteq B_{j}} \frac{m\left(B_{j}\right)}{\left|B_{j}\right|}
$$

where $B_{j} \in 2^{\Theta}$, is the focus element and $\left|B_{j}\right|$ is the number of elements in subset $B_{j}$.

\subsection{Definition of fuzzy probability masses}

The definition of the probability masses is the most crucial step in the application of Dempster-Shafer evidence theory [17]. In this study, we establish a new BPA function to fulfil the classification purpose, in which each feature distinguishes two complementary subsets, $\mathrm{A}$ and $\mathrm{B}$, and the frame of discernment is $\{\varnothing, A, B, \mathrm{~A} \cup \mathrm{B}\} . \mathrm{m}(\mathrm{A} \cup \mathrm{B})=1-\mathrm{m}(\mathrm{A})-\mathrm{m}(\mathrm{B})$. Probability masses are obtained by defining a BPA function $\mathrm{P}_{\mathrm{Ai}}(\mathrm{x})$ for $\mathrm{A}$, and $\mathrm{P}_{\mathrm{Bi}}(\mathrm{x})$ for $\mathrm{B}$, respectively, hence each data source $i$ with $m_{i}(A)=P_{A i}(x)$ and $m_{i}(B)=P_{B i}(x) . P_{A i}(x)$ is interpreted as the probability of an image pixel belonging to class $A$ when the output of source $i$ is $x$. It can also be interpreted as the result of an initial classification using only source $\mathrm{i}$ to distinguish classes $\mathrm{A}$ and $\mathrm{B}$. The distinguished complementary subsets for each feature in this work are shown in Table 1, where B stands for building, $\mathrm{G}$ for grass, $\mathrm{R}$ for road, and $\mathrm{T}$ for tree.

Table 1. Complementary subsets for each feature

\begin{tabular}{ccc}
\hline \multirow{2}{*}{ Features } & \multicolumn{2}{c}{ Classes } \\
\cline { 2 - 3 } & $A$ & $B$ \\
\hline FE & BUT & GUR \\
HD & $\mathrm{T}$ & BUGUR \\
IN & BUGUR & T \\
NDVI & TUG & BUR \\
\hline
\end{tabular}




$$
\begin{aligned}
& P_{A_{i}}(x)=\left\{\begin{array}{cl}
P_{2} & x<h_{1} \\
P_{2}-\frac{P_{2}-P_{1}}{h_{12}-h_{1}}\left(x-h_{1}\right) & h_{1}<x \leq h_{12}
\end{array}\right. \\
& P_{B_{i}}(x)= \begin{cases}P_{1}+\frac{P_{2}-P_{1}}{h_{2}-h_{12}}\left(x-h_{12}\right) & h_{12}<x<h_{2} \\
P_{2} & x \geq h_{2}\end{cases} \\
& P_{A \cup B_{i}}(x)=\left\{\begin{array}{cl}
P_{1} & x \leq h_{1} \\
P_{1}+\frac{P_{2}-P_{1}}{h_{12}-h_{1}}\left(x-h_{1}\right) & h_{1}<x \leq h_{12} \\
P_{2}-\frac{P_{2}-P_{1}}{h_{2}-h_{12}}\left(x-h_{12}\right) & h_{12}<x<h_{2} \\
P_{1} & x \geq h_{2}
\end{array}\right.
\end{aligned}
$$

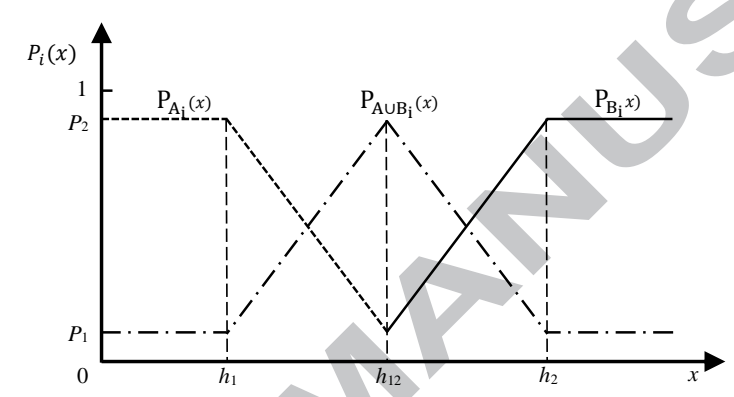

Figure 2. Curves of the BPA function

In this study, the BPA function is built empirically as expressed in Equations (5) to (7). The curves of the BPA function are shown in Figure 2. Unlike the original BPA function used in [12] and [13], we define a new class $A \cup B$ to represent the fuzzy class between classes $A$ and $B$, and it is not certain which class the elements of class $A \cup B$ belong to. $P_{A \cup B_{i}}(x)$ is interpreted as the probability of an image pixel belonging to class $A \cup B$ when the output of source $i$ is $x$ [18]. As shown in Figure 2, if the input $\mathrm{x}$ is less than the threshold $\mathrm{h}_{1}$, we can assume that the assignment of a pixel to class $A$ or class $A \cup B$ is almost certain, and we can obtain $\mathrm{P}_{\mathrm{A}_{\mathrm{i}}}(\mathrm{x})$ and $\mathrm{P}_{\mathrm{A \cup B}_{\mathrm{i}}}(\mathrm{x})$ according to Equations (5) to $(7)$. $\mathrm{P}_{\mathrm{B}_{\mathrm{i}}}(\mathrm{x})$ is zero as it represents the probability of class $B$. The equation of $\mathrm{P}_{\mathrm{A} \cup \mathrm{B}_{\mathrm{i}}}(\mathrm{x})=1-$ $\mathrm{P}_{\mathrm{A}_{\mathrm{i}}}(\mathrm{x})-\mathrm{P}_{\mathrm{B}_{\mathrm{i}}}(\mathrm{x})$ which can also be expressed as $\mathrm{m}(\mathrm{A} \cup \mathrm{B})=1-\mathrm{m}(A)-\mathrm{m}(B)$ is satisfied. If the input $\mathrm{x}$ is greater than the threshold $\mathrm{h}_{2}, \mathrm{P}_{\mathrm{A}_{\mathrm{i}}}(\mathrm{x})$ is zero and we can have $\mathrm{P}_{\mathrm{B}_{\mathrm{i}}}(\mathrm{x})$ and $\mathrm{P}_{\mathrm{A} \cup \mathrm{B}_{\mathrm{i}}}(\mathrm{x})$ worked out according to Equations (5) to (7).

In Figure 2, the information from a sensor should never be assumed to be $100 \%$ certain. Therefore we use $\mathrm{P}_{1}=2 \%, \mathrm{P}_{2}=98 \%$ as the lower and upper boundaries in practice to reflect this uncertainty. We call the proposed BPA function as a "Fuzzy BPA Function", while the DS method with a fuzzy BPA function is expressed as F-DS. The advantages of the fuzzy BPA function are listed as follows:

(i) It is reliable to classify those pixels whose values reside between $h_{1}$ and $h_{2}$ into a fuzzy category which is a new fuzzy class belonging neither to $A$ nor to $B$. 
(ii) This method reduces the degree of uncertainty in data processing with the fuzzy class and it can take the advantages of DS theory to deal with the uncertainty problem better compared with the simple DS method.

\subsection{Median filtering}

When we classify image pixels with a BPA function, it is observed that there are isolated pixel points scattered in an area representing another class. For example, when scattered "road" pixel points reside among a grass area, these pixel points should be eliminated to reduce classification errors [19]. It is our initiative to apply filtering in the interim result in the DS combination process for performance improvement. Considering the nature of the noise, we have attempted both median filtering and contraharmonic mean filtering [20, 21]. Based on their performance and the computational cost, median filtering is chosen to tackle the problem.

In this study, the targets of median filtering process are the probability images obtained by BPA functions, as shown in Figure 1.

\subsection{Hierarchical structure}

The two-stage hierarchical structure is considered based on an observation that when using HD feature to distinguish trees from other classes, there is misclassification due to dense leave areas and tree stems where the LIDAR penetration fails [22]. Moreover an edge of building is easily confused with the trees in the HD feature [23]. Interestingly LIDAR intensity, the IN feature, is complementary to the HD feature in this sense. In the study, the combination of HD and IN dedicates to Stage 1 in tree and non-tree classification, and stage 2 accepts the output from Stage 1 as its input, joined with other two features FE and NDVI for further classification. This two-stage hierarchical structure effectively combines features with different characteristics in land cover classification for the four designated classes. It aims to reduce classification errors hence to improve the classification accuracy. The proposed method with both the fuzzy BPA function and the hierarchical structure is called F-DS-FH in the later discussion.

\section{Experiment and discussion}

\subsection{Experiments and results}

The proposed method given in Sections 2 and 3 is evaluated by experiments in order to verify its performance. MATLAB R2013a is chosen as the software platform for the testing experiments and result analysis. Two data sets used in the tests are shown in Figure 3 and Figure 5, respectively, with carefully established ground truth. The parameters of the BPA function are set empirically as shown in Table 2 . The window size in median filtering is $3 \times 3$ in the first stage and $5 \times 5$ in the second stage as these window sizes tend to deliver the best result.

Table 2. Parameters of BPA function

\begin{tabular}{cccc}
\hline & $\mathrm{h}_{1}$ & $\mathrm{~h}_{12}$ & $\mathrm{~h}_{2}$ \\
\hline HD & 76.00 & 136.00 & 178.50 \\
IN & 72.00 & 138.00 & 180.00 \\
FE & 51.00 & 70.00 & 122.40 \\
NDVI & 102.00 & 132.00 & 200.00 \\
\hline
\end{tabular}


In the experiments, four classification methods were compared on two testing data sets. They are: (a) simple DS evidence theory (S-DS), (b) DS evidence theory with a fuzzy BPA function (F-DS), (c) DS evidence theory with medium filtering and a fuzzy BPA function (F-DS-F), and (d) the proposed hierarchical approach (F-DS-FH). Based on the observations stated in Section 3.4 that feature IN complements feature HD in tree identification, the hierarchical F-DS-FH is designed and feature IN is specifically used to support HD for distinguishing trees from other classes in the F-DS-FH. The classification results on the two testing datasets of the four methods with the ground-truth are shown in Figure 4 and Figure 6, respectively.

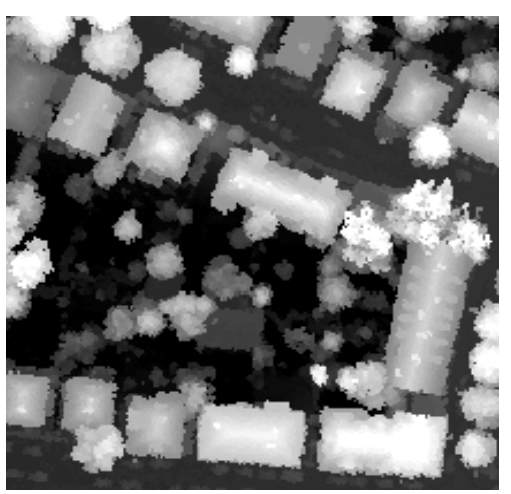

(a)

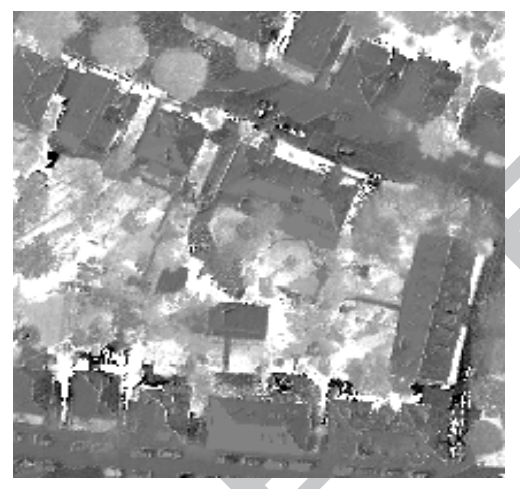

(d)

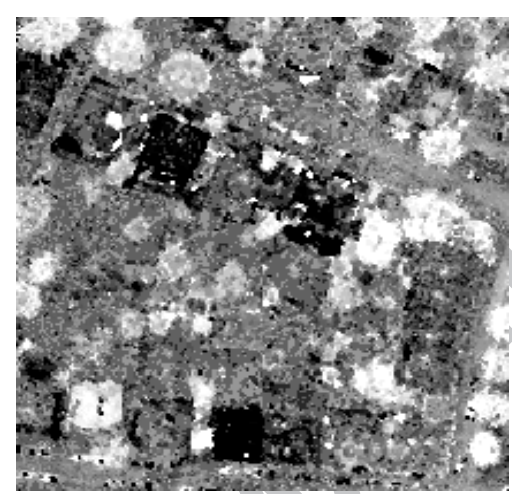

(b)

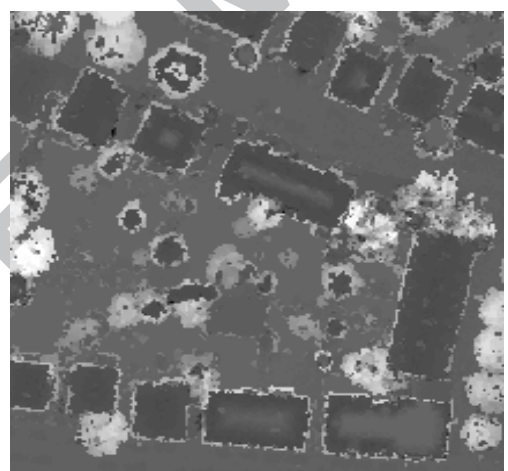

(e)

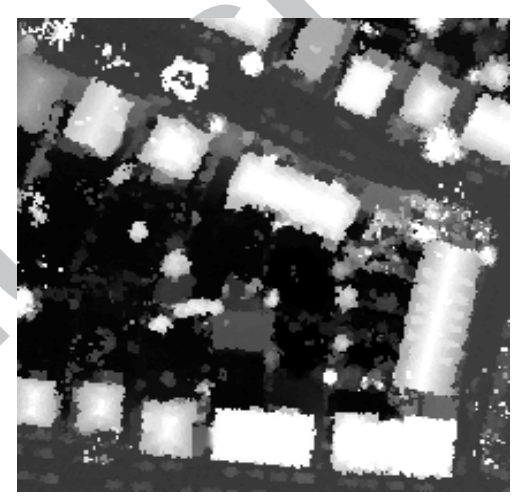

(c)

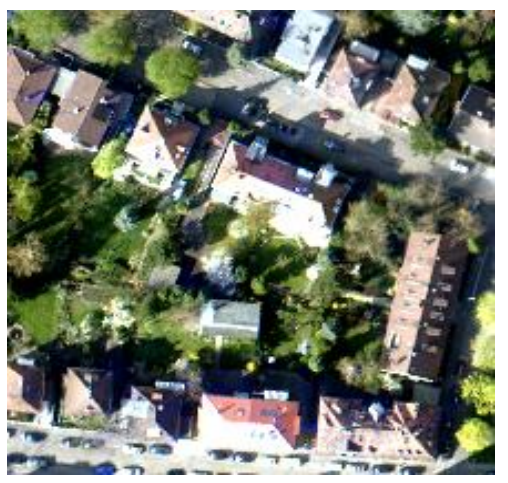

(f)

Figure 3. The initial data used in Test 1. (a) FE, (b) IN, (c) LE, (d) NDVI, (e) HD, (f) RGB image

\subsection{Evaluation of the results and discussion}

For the results demonstrated in both Figure 4 and Figure 6, we use "black" color for building, "dark grey" for trees, "light grey" for grass, and "white" for road. The results of Test 1 in Figure 4 show that, from the methods of S-DS to F-DS-FH, the classification accuracy increases when compared to the ground-truth. Two observations which favor the F-DS-FH method are explained as follows.

(1) As can be seen from the S-DS and F-DS results, the most confusing groups are trees and buildings. A large number of black points which should have been classified as trees was misclassified as buildings. This is because laser beams may not penetrate in the dense leaf cover and tree stem areas. This made HD failed to distinguish trees from other classes, especially buildings in the scene. The IN feature is the intensity of the laser reflection. The F-DS-FH method takes advantage of the complementary information provided by HD and IN for tree identification, and synthesizes them in the hierarchical framework. It significantly 
reduced the isolated black points in the tree area, and the corresponding result closely reflects the ground-truth.

(2) Another pair of classes which are easily confused is "road" and "grass" classes. This occurs as there are small bare areas between blades of grass. Under this circumstance, the pixel-based classification made the NDVI feature not working to connect a grass area as a solid connected component. The results, as we can see from Figure 4(a) the S-DS image and Figure 4(b) the F-DS image, have shown that "road" class points scattered in grass areas and vise verse. Median filtering is able to eliminate these scattered noise pixels and treat grass areas as a solid connected component. As a result, in the F-DS-F and F-DS-FH images which are demonstrated in Figures 4(c) and 4(d), respectively, the scattering points are largely eliminated.

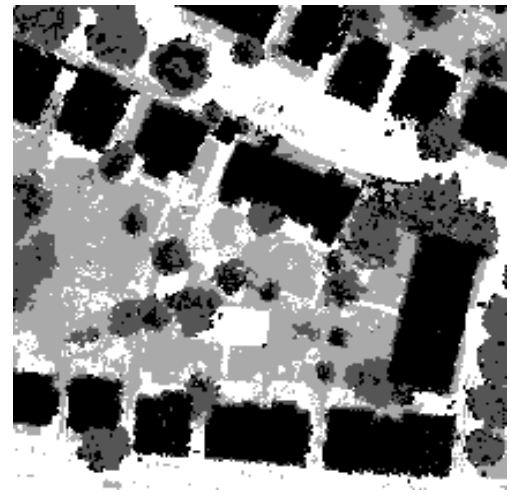

(a)

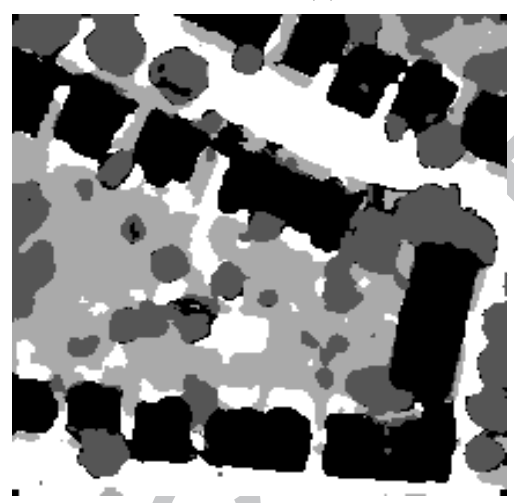

(d)

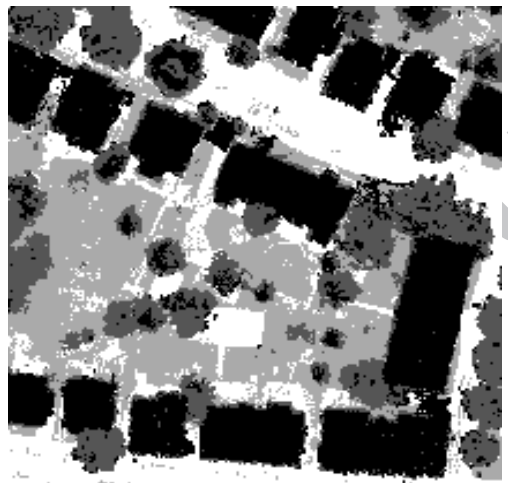

(b)

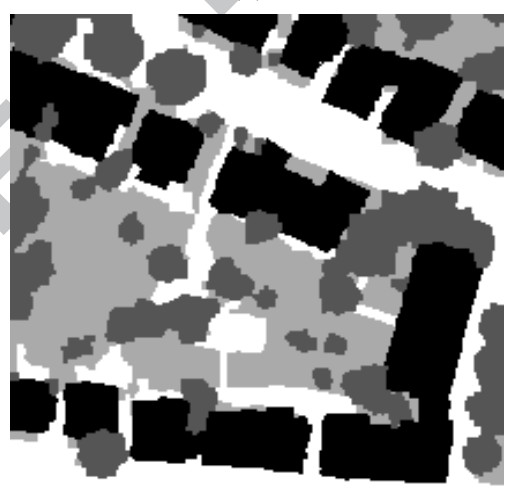

(e)

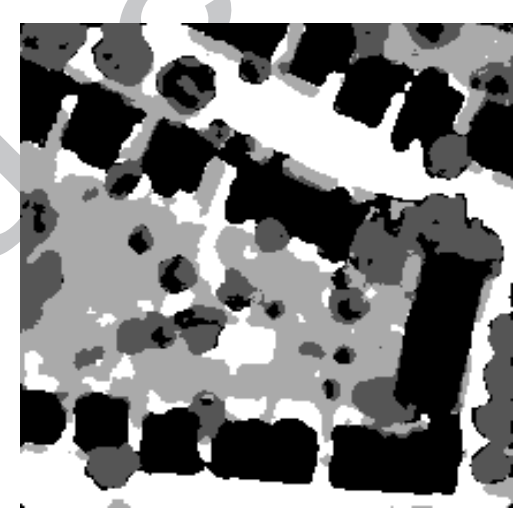

(c)

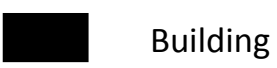

Grass

Road

Tree

Figure 4. The classification results of Test 1. (a) S-DS, (b) F-DS, (c) F-DS-F, (d) F-DS-FH, (e) Ground-truth

Table 3 presents the classification accuracy of Test 1 . The data shown in the table represent the percentage of the pixel number correctly classified by the corresponding method in a class with comparison to the relevant pixel number in the ground-truth. We can see from Table 3 that F-DS-FH method has achieved $83.95 \%$ accuracy in the "tree" class, much higher than the accuracy of $68.18 \%$ obtained by using the S-DS method or the $72.48 \%$ obtained with the F-DS-F method. It proves that the hierarchical framework of F-DS-FH method has higher competence in distinguishing the "tree" class from other classes. Comparison of the average classification accuracy values for each method also shows that the F-DS-FH method gives the best average classification accuracy. Table 3 also gives the average growth rate based on S-DS for classification accuracy. 
Table 3. Classification accuracy (\%) on test data set 1

\begin{tabular}{llllllr}
\hline & building & tree & Grass & Road & Average & average growth rate \\
\hline S-DS & 86.07 & 68.18 & 84.28 & 87.24 & 81.81 & 0 \\
F-DS & 87.33 & 72.85 & 87.23 & 85.70 & 83.59 & 2.17 \\
F-DS-F & $\mathbf{9 2 . 5 5}$ & 72.48 & $\mathbf{9 1 . 3 3}$ & $\mathbf{8 8 . 0 8}$ & 86.58 & 5.83 \\
F-DS-FH & 91.77 & $\mathbf{8 3 . 9 5}$ & 89.50 & 87.96 & $\mathbf{8 8 . 5 4}$ & 8.22 \\
\hline
\end{tabular}

In addition, both classification accuracy and computational time of the experiment were compared with the result obtained in [12]. An indirect comparison was conducted, in which the growth rate of classification accuracy to the S-DS is used. The EBF-MRF method proposed in [12] shows increases in accuracy of $6.62 \%$ compared to the S-DS method, while the accuracy of the F-DS-FH method developed in this study shows an increase in accuracy of $8.22 \%$ compared with the S-DS method.

The computational time of the F-DS-FH method proposed in this paper is also compared with the time required for the methods reported in [12], as shown in Table 4. From the table we can see that the fastest method considered in [12] is the ICM-MRF method, with a computational time of 13.16 second, but F-DS-FH method proposed in this paper only required $0.65 \mathrm{~s}$. This shows that, with the non-iterative nature, the F-DS-FH method not only achieves better accuracy but also more efficient for land-cover classification with LIDAR data and co-registered NIR and RGB images.

Table 4. Comparison of the computational time in Test 1 (image size: $220 * 220$ )

\begin{tabular}{c|c|cccc}
\hline \multicolumn{3}{|c}{ Method } & \multicolumn{3}{c}{ Methods in [12] } \\
\cline { 1 - 1 } Method & F-DS-FH & ICM-MRF & SA-MRF & BP-MRF & EBP-MRF \\
\cline { 1 - 1 } $\begin{array}{c}\text { computational } \\
\text { time (second) }\end{array}$ & $\mathbf{0 . 6 5}$ & 13.16 & 38572.49 & 468.4 & 127.85 \\
\hline
\end{tabular}

Test 2 was carried out in order to verify the robustness of proposed method. The size of the data used in Test 2 is $200 \times 200$, and the data and results are shown in Figure 5 and Figure 6, respectively.

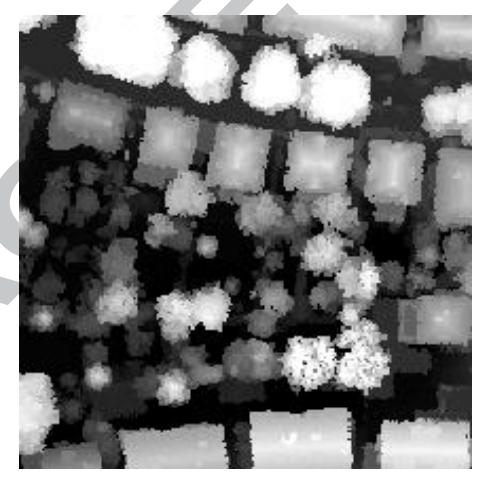

(a)

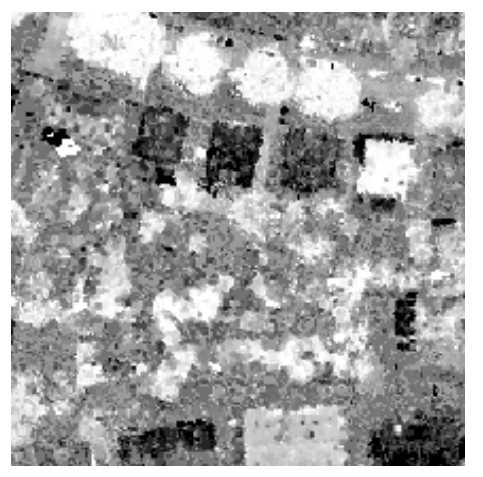

(b)

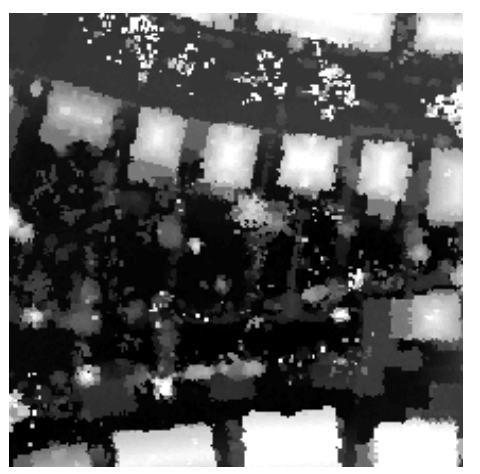

(c) 


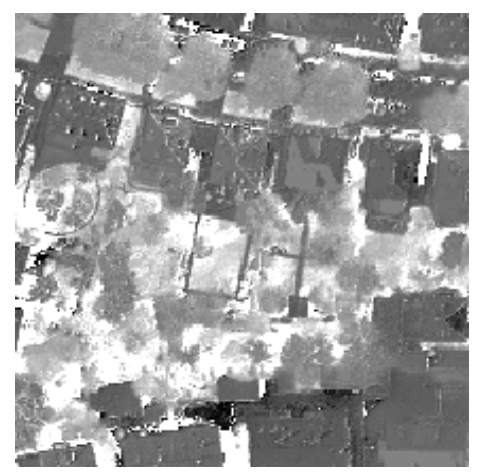

(d)

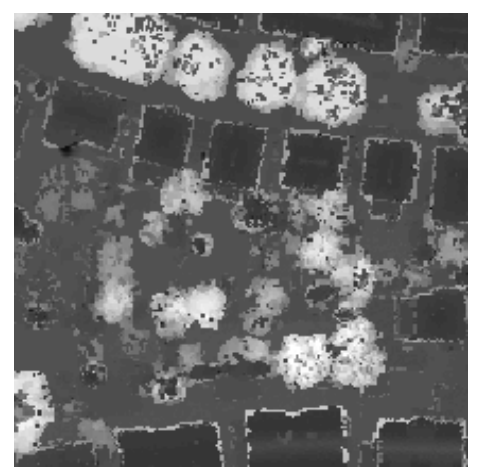

(e)

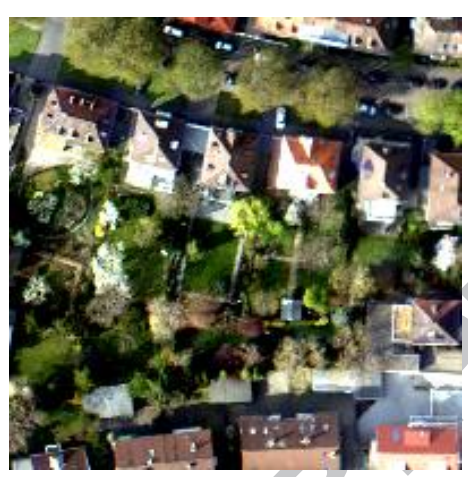

(f)

Figure 5. The initial data used in Test 2. (a) FE, (b) IN, (c) LE, (d) NDVI, (e) HD, (f) RGB image

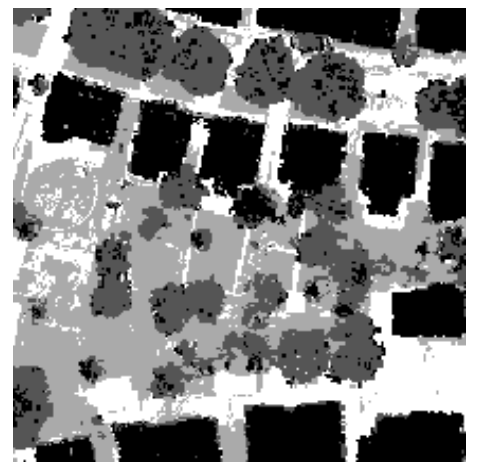

(a)

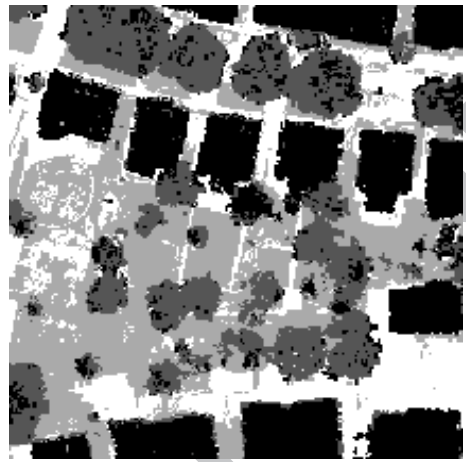

(b)

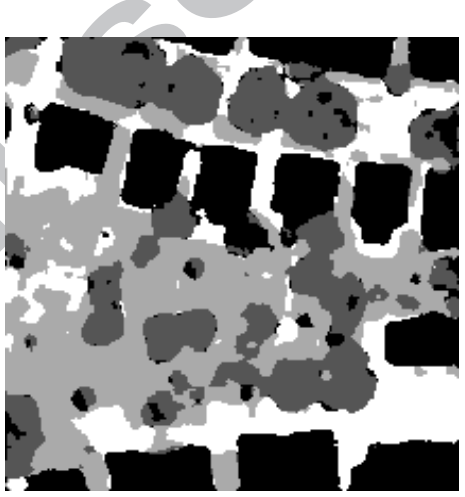

(c)

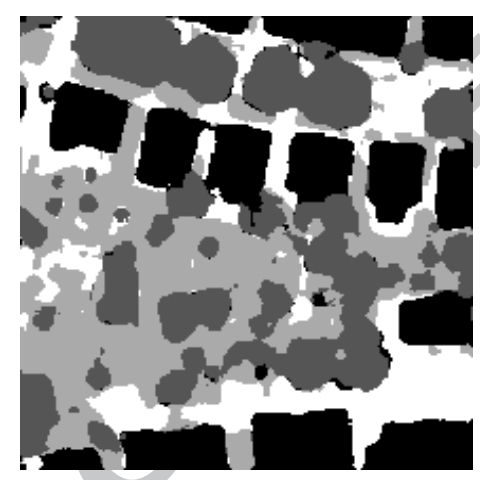

(d)

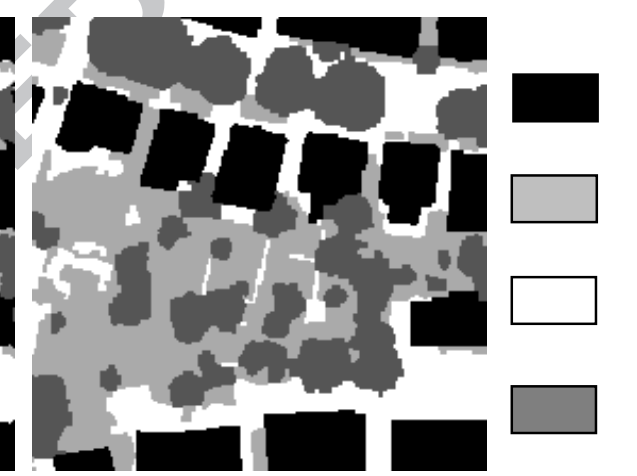

Building

Grass

Road

Tree

Figure 6. The classification results of test 2. (a) S-DS, (b) F-DS, (c) F-DS-F, (d) F-DS-FH, (e) Ground-truth

The classification accuracy and computational time of Test 2 are presented in Table 5 and Table 6 , respectively.

Table 5. Classification accuracy (\%) of Test 2

\begin{tabular}{llllllc}
\hline & building & tree & grass & Road & average & average growth rate \\
\hline S-DS & 90.89 & 75.44 & 88.07 & 85.03 & 84.83 & 0 \\
F-DS & 90.41 & 79.58 & 89.29 & 83.02 & 85.71 & 0.88 \\
F-DS-F & $\mathbf{9 4 . 5 6}$ & 82.77 & $\mathbf{9 3 . 4 6}$ & $\mathbf{8 3 . 7 3}$ & 88.90 & 4.07 \\
F-DS-FH & 93.78 & $\mathbf{9 2 . 9 0}$ & 87.74 & 83.29 & $\mathbf{8 9 . 7 1}$ & 5.76 \\
\hline
\end{tabular}


Table 6. Comparison of the computational time in Test 2 (image size: 200*200)

\begin{tabular}{c|c|cccc}
\hline \multicolumn{3}{c}{ Method } & \multicolumn{3}{c}{ Methods in reference [12] } \\
\cline { 1 - 1 } method & F-DS-FH & ICM-MRF & SA-MRF & BP-MRF & EBP-MRF \\
\cline { 1 - 1 } $\begin{array}{c}\text { computational } \\
\text { time (second) }\end{array}$ & 0.19 & 21.65 & 40841.08 & 786.53 & 199.84 \\
\hline
\end{tabular}

\section{Conclusions}

Focused on classification in which there is no training sample available, this study employed the Dempster-Shafer evidence theory, and established a combination framework in the hierarchical structure for land-cover classification for urban areas, where building, tree, road and grass field dominate land covers. The proposed method has been tested on two datasets with LIDAR first echo (FE), last echo (LE) and intensity (IN) fused with co-registered near infrared (NIR) and color images. Compared to other methods $[12,24]$, the proposed framework is superior in both computational efficiency and classification accuracy. Confirmed by the experimental results, not only the computational time is dramatically reduced but also the classification accuracy is significantly improved. It has achieved results that match or are better than results by other state-of-the-arts methods [4]. The novelty and the main contributions of this study are summarized in the following points:

(1) It is a non-iterative approach and doesn't require training processes. Therefore it can be used in applications in which it is difficult to acquire training samples and it requires a rapid response to urban area land cover information.

(2) Instead of using a linear mass function in distributing the probability to each class, a fuzzy class was established with a newly developed BPA function to deal with pixel points residing in a region mixed with two classes. This is an effective way to reduce misclassification. It takes advantage of the DS evidence theory and plays a better role than the original DS theory.

(3) Noise inevitably introduced during the probability distribution processing was taken into account in the intermediate stages. We applied median filtering to probability image de-noise. This is an entirely new idea that has never been seen in the previous studies. Median filtering is effectively employed to these interim probability images after the probability distribution processing. This has significantly reduced the error rate, and largely improved the classification accuracy.

(4) In the proposed combination framework, complementary features HD and IN have been jointly used to contribute to tree identification in the scene based on observations to the effects introduced by the original data. A hierarchical structure was designed to implement the process. This makes the classification results more reliable, especially for tree identification.

Interesting findings have been presented in the paper. These findings inspired us to build the effective framework in which the hierarchical structure was created to incorporate the IN feature in the feature space and filtering processes were applied to interim results for performance improvement. The experimental results have shown that the proposed method works well in urban area land cover classification. The average classification accuracy is up to $88.54 \%$, and it is $8.22 \%$ higher than that with the basic DS method. Compared with the most accurate iterative method EBF-MRF proposed in [12], our method has not only improved the classification accuracy of about $1.60 \%$, but also reduced the computational time from 127.85 seconds to 0.65 seconds on the first testing area, and from 199.84 seconds to 0.19 seconds on the second testing area. This has shown the 
significant improvement in computational efficiency.

\section{Acknowledgements}

This paper is supported by the National Natural Science Foundation of China (No. 61171057) and the Bai Ren Programme of Shan Xi Province. The authors would like to thank TopoSys GmbH (renamed as Trimble Holdings $\mathrm{GmbH}$ ) for the data supply.

\section{References}

[1] M A Wulder, J C White, R F Nelson, et al. (2012), LiDAR sampling for large-area forest characterization: a review, Remote Sensing of Environment, 121: 196-209.

[2] A A Matkan, M Hajeb, S Sadeghian (2014), Road extraction from Lidar using support vector machine classification, Photogrametric Engineering \& Remote Sensing, 80(5): 409-422.

[3] R Wang (2013), 3D building modeling using images and LiDAR: A review. International Journal of Image and Data Fusion. 4(4):273-292.

[4] W Y Yan, A Shaker, N EI-Ashmawy (2015), Urban land cover classification using airborne LiDAR data: A review. Remote Sensing of Environment. 158:295-310.

[5] J Niemeyer, J D Wegner, C Mallet, et al. (2011), Conditional random fields for urban scene classification with full waveform LiDAR data. Photogrammetric Image Analysis, Lecture Notes in Computer Science, 6952:233-244.

[6] G Mountrakis, J Im, C Ogole (2011), Support vector machines in remote sensing: A review. ISPRS Journal of Photogrammetry and Remote Sensing, 66(3): 247-259.

[7] G Li, N Chehata, C Mallet, et al. (2011), Relevance of airborne LiDAR and multispectral image data for urban scene classification using random forests. ISPRS Journal of Photogrammetry and Remote Sensing. 66(1):56-66.

[8] O S Ahmed, S E Franklin, M A Wulder, and J C White (2015), Characterizing stand-level forest canopy cover and height using Landsat time series, samples of airborne LiDAR, and the Random Forest algorithm, ISPRS Journal of Photogrammetry and Remote Sensing, 101:89-101

[9] G Xu, X Zhu, D Fu, J Dong, and X Xiao (2017), Automatic land cover classification of geo-tagged field photos by deep learning, Environmental Modelling \& Software, 91:127-134

[10] B Chen, B Huang, and B Xu (2017), Multi-source remotely sensed data fusion for improving land cover classification, ISPRS Journal of Photogrammetry and Remote Sensing, 124:27-39

[11] D R Pereira, and J P Papa (2016), A new approach to contextual learning using interval arithmetic and its applications for land-use classification, Pattern Recognition Letter, 83:188-194

[12] Y Cao, H Wei, H J Zhao, et al. (2012), An effective approach for land-cover classification from airborne Lidar fused with co-registered data, International Journal of Remote Sensing, 33:5927-5953.

[13] F Rottensteiner, J Trinder, S Clode, et al. (2005), Using the Dempster-Shafer method for the fusion of LIDAR data and multi-spectral images for building detection. Information Fusion. 6:283-300.

[14] G Bell (2011), Tree Identification Using Fusion of Images and LIDAR Data, The University of Reading for the degree of Master of Science in Cybernetics

[15] F B Yang, X X Wang (2010), Combination Method of Conflictive Evidences in D-S Evidence 
Theory, Beijing: National Defense Industry Press, 148-153.

[16] K X Qiao, S B Yin, and S J Qu (2012), Multi-scale fused edge detection algorithm based on conflict redistribution DSmT, Journal of Computer Applications, 32(4): 1050-1052.

[17] P P Feng, F B Yang, and H Wei, et al.(2015),-A fast method for land-cover classification from LIDAR data based on hybrid Dezert-Smarandache Model, ICIC-ELB, 6(11):3109-3114.

[18] X T Li, F B Yang, X Y Zhou, et al. (2012), Missile defence combat system situation analysis based on the theory of possibility, Computer Development and Applications, 25(3): 6-9.

[19] Z G Liu (2011), Method for road information extraction based on LIDAR technology, Journal of Transport Information and Safety, 29(2): 121-124.

[20] R C Gonzalez and R E Woods (2002), Digital Image Processing, $2^{\text {nd }}$ Edition, Prentice-Hall Inc

[21] O Marques (2011), Practical Image and Video Processing Using MATLAB, Wiley-IEEE Press

[22] J P O'Neil-Dunne, S W MacFaden, and A R Royar, et al. (2013), An object-based system for LiDAR data fusion and feature extraction, Geocarto International, 28: 227-242.

[23] X G Liu, J Zhang, and W Gao (2006), Extract buildings quickly from LIDAR point cloud data, Earth Science-Journal of China University of Geosciences, 31(5):615-618.

[24] W Essa, B Verbeiren, J van der Kwast, et al. (2012). Evaluation of the DisTrad thermal sharpening methodology for urban areas, International Journal of Applied Earth Observation and Geoinformation, 19:163-172. 